\title{
Gerontopsychiatrie - Traditionelle Handlungsmuster modifizieren
}

Gerontopsychiatrie ist einmal mehr Schwerpunktthema der Zeitschrift. Nicht allein der stetige Wissenszuwachs auf dem Gebiet der psychischen Störungen im höheren Lebensalter ruft diese Themenwahl hervor; mittlerweile hat auch eine intensive Überprüfung von Nutzen und Risiken vorhandener Therapieoptionen begonnen, die im einen oder anderen Bereich gerontopsychiatrischen Handelns zu einer Modifizierung traditioneller Handlungsmuster geführt hat oder noch führen wird. Hierzu wollen die folgenden drei Arbeiten einen Beitrag leisten.

R. Perneczky, München, zeigt bei den frontotemporalen Demenzen die Symptomvielfalt auf, ihre Unterschiede zur Symptomatik der Alzheimer Demenz und die erheblichen psychosozialen Konsequenzen der Verhaltensstörungen bei dieser Demenzform, beispielsweise im rechtlichen Bereich oder bei der Fahrtüchtigkeit. Hierauf liegt der Schwerpunkt seiner Betrachtung, gleichwohl werden therapeutische Aspekte dieser im wesentlichen schwer behandelbaren Demenz vorgestellt.

L. Drach, Schwerin, verhilft dem Leser in seiner Darstellung zum Anschluss an den aktuellen Kenntnisstand über die Demenz mit LewyKörperchen. Er macht in der Schilderung von Symptomen und diagnostischen Spielregeln auf die neuere internationale Entwicklung der Kriterien zur Erkennung dieser Demenz auf- merksam, die von früheren diagnostischen Kriterien aus den 90er-Jahren zum Teil entscheidend abweichen. Zudem vermittelt er dem Leser Einblicke in die neuropathologischen Kennzeichen zur Abgrenzung von Alzheimer-Demenz, Parkinson Demenz und Demenz mit Lewy-Körperchen. Der Abschnitt zu den therapeutischen Optionen ist stark pharmakologisch fokussiert und verweist auf Antidementiva vom Typ der Cholinesterasehemmer und speziell ausgewählte neuere Neuroleptika als angemessene und wirksame Mittel der Behandlung.

B. Ibach, Neuss, führt den Leser zum gegenwärtigen Kenntnisstand der differenziellen neuroleptischen Behandlung der Verhaltensstörungen bei Demenz, insbesondere bei AlzheimerDemenz. Nach eingehender Darstellung der kontrollierten klinischen Studien zur Wirksamkeit der neueren Neuroleptika (Neuroleptika der zweiten Generation: second generation antipsychotics: SGA) widmet er sich ausführlich der Problematik der Arzneimittelsicherheit. Hierbei kommt er am Beispiel der Diskussion über das Risiko zerebrovaskulärer Ereignisse insgesamt zu dem Schluss, dass neuere und herkömmliche Neuroleptika Risiken tragen, herkömmliche Neuroleptika aber tendenziell mit einem höheren Risiko behaftet sind. Ein Diskussionsteil mit praktischen Handlungsempfehlungen für den in der Praxis tätigen Arzt rundet den Beitrag ab.

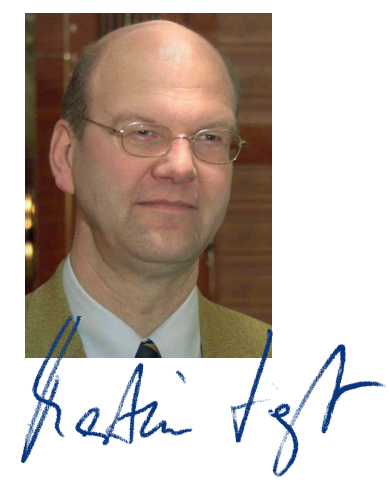

PD Dr. Martin Haupt, Düsseldorf 